Journal of Computer Science 8 (2): 259-264, 2012

ISSN 1549-3636

(C) 2012 Science Publications

\title{
A New Ventricular Late Potential Classification System Using Ant Colony Optimization
}

\author{
${ }^{1}$ Sankara Subramanian, A., ${ }^{2}$ G. Gurusamy and ${ }^{3}$ G. Selvakumar \\ ${ }^{1}$ Department of Electrical Engineering, \\ NPAC Polytechnic College, Kotagiri-643217, India \\ ${ }^{2}$ Bannari Amman Institute of Technology, Sathy, India \\ ${ }^{3}$ Department of Electrical Sciences, \\ Annapoorani College of Engineering Salem, 636308, India
}

\begin{abstract}
Problem statement: Ventricular Late Potentials (VLPs) are low-level high frequency signals that are usually found within the terminal part of the QRS complex from patients after myocardial infarction. Patients with VLPs are at risk of developing ventricular tachycardia, which is the major cause of death if patients suffering from heart disease. Approach: Discrete Wavelet Transform was used to detect VLPs and then ANT Colony Optimization (ACO) was applied to classify subjects with and without VLPs. Results: A set of Discrete Wavelet Transform (DWT) coefficients was selected from the wavelet decomposition. Three standard parameters of VLPs such as $\mathrm{QRS}_{\mathrm{T}}, \mathrm{D}_{40}$ and $\mathrm{V}_{40}$ were established. After that a novel clustering algorithm based on Ant Colony Optimization was developed for classifying arrhythmia types. Conclusion: The wavelet decomposition enabled us to perform the task efficiently and produced reliable results.
\end{abstract}

Key words: Discrete wavelet transforms, ventricular late potentials, wavelet decomposition, Ant Colony Optimization (ACO), Signal-Averaged Electrocardiograms (SAECGs)

\section{INTRODUCTION}

The Ventricular Late Potentials (VLPs) are low amplitude, high frequency signals that generally occur within the terminal part of the QRS complex and the beginning of the ST segment. VLPs represent delayed conduction through the ventricular myocardium. They can be thought of as small action potentials from myocytes isolated by fibrosis that depolarize late, after the majority of myocytes that constitute the QRS complex have depolarized. VLPs appear as fractioned signals with irregularity in shape on the body surface. From the studies in clinical cardiology, it has been shows that the occurrence of VLP is prevalent in post Myocardial Infarction (MI) patients at risk of developing Ventricular Tachycardia which is one of the leading causes of sudden cardiac death (Mozus et al., 2011).

Hence the detection of VLPs has become a topic of interest in clinical cardiology for over three decades. Early diagnosis of heart disease by detecting VLPs might save a large number of lives. The recordings of VLPs are usually made on body surface by signal averaging techniques applied to ST segment.
Ventricular late potentials are obtained from SignalAveraged Electrocardiograms (SAECGs).

The study presented here investigates the application of the wavelet transform for the detection of VLPs. The wavelet transform is a powerful tool which is able to characterize the ECG signals both in time and in frequency domains thus allowing a better insight in the pathological phenomenon of VLPs. The Discrete Wavelet Transform (DWT) has been used for analyzing, decomposing and compressing the ECG signals. The wavelet transforms make possible, the decomposition of a signal into a set of different signals of restricted frequency bands.

Wavelet processing can be considered as a set of band pass filters. Moreover, the discrete wavelet transform corresponds to a multi-resolution analysis which can reduce the redundancy of each filtered signal so that the processing algorithm can be applied effectively to a small data subset of the original signal.

After that a novel classification algorithm based on ACO is developed for classifying arrhythmia types. The system consists of three stages and is constructed as shown in Fig. 1. The first stage of learning phase

Corresponding Author: Sankara Subramanian, A., Department of Electrical Engineering, NPAC Polytechnic College,

Kotagiri-643217, India 
involves extraction of the feature values. The second stage involves the storage of information over Relational Data base Management System (RDBMS). The last stage involves the clustering of QRS parameters using the ACO technique to construct arrhythmia classes. Test or control phase is used to control algorithm's efficiency and correctness as shown in Fig. 2. The first stage of testing phase is parameter extraction. Previously built cluster information serves as an input to the testing system.

In this study, a set of data containing records for both Ventricular Tachycardia (VT) and non-VT myocardial infarction patients is used. Each record is decomposed into a number of time signals with different frequency contents using DWT techniques. The DWT level containing the frequency range of interest in each signal is then used in the analysis.

Ventricular late potentials: The frequency distributions of ECG signals are classified as lower frequency $\mathrm{P}$ and $\mathrm{T}$ waves, middle-to-high frequency QRS complex and high frequency late potentials when they exist. The $\mathrm{P}$ and $\mathrm{T}$ waves are Medium Amplitude Low Frequency Signals (MALFS), the QRS complex is High Amplitude Medium Frequency Signal (HAMFS) and the VLP is a Low Amplitude High Frequency Signal (LAHFS). Figure 3 shows the different frequency components of ECG presented against their respective amplitudes.

Characteristic changes in these waves are an indication of possible abnormalities. Late in the ECG cycle, when high frequency events occur, these low amplitude signals are identified as late potentials. VLPs Have been shown to be a predictive of arrhythmia of the heart. When arrhythmias, such as ventricular tachycardia do occur, the QRS undergoes important morphological changes. These changes may be in the form of widening of the QRS complex. As the QRS widens, its power spectra shows diminished contributions at higher frequencies and these are spread out over a wider body of the signal. This empirical description of time domain features of the ECG signal lends itself particularly well to analyze it by timefrequency and time-scale methods. In previous studies, low amplitude, high frequency signal in the last $40 \mathrm{~ms}$ of the filtered QRS and a prolonged QRS duration have been shown to identify patients with ventricular tachycardia. A late potential is defined as a low amplitude signal $20 \mu \mathrm{V}$ in the last $40 \mathrm{~ms}$ of the filtered QRS complex and a long filtered QRS complex is defined as total filtered QRS duration greater than 120 $\mathrm{m}$ sec where filtering is carried out using the wavelet transform (Tirtom et al., 2008).
Signal Averaged ECG (SAECG): Lead systems: For recording VLPs from the surface of the body most investigators use an XYZ lead system formed by three orthogonal bipolar electrode combinations. Signals from the three bipolar electrodes can be combined into a spatial vector magnitude which is given by Eq. 1, yielding a composite waveform. Others have used a variety of primordial lead systems to achieve closer proximity to the left ventricle.

Some have suggested that a primordial lead system has advantages (Sivakumar et al., 2011); others have shown there is no advantage (Zandi and Moradi, 2006). Most current systems use orthogonal, bipolar XYZ ECG leads, which are recorded, averaged, filtered and combined into a vector magnitude called the filtered QRS complex.

Amplification: ECG electrode signals are initially amplified 10-100 times with a wide-frequency band pass before analog to digital conversion. Some investigators further amplify the signals after analog to digital conversion.

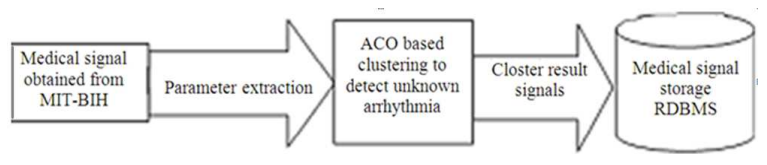

Fig. 1: Training system model

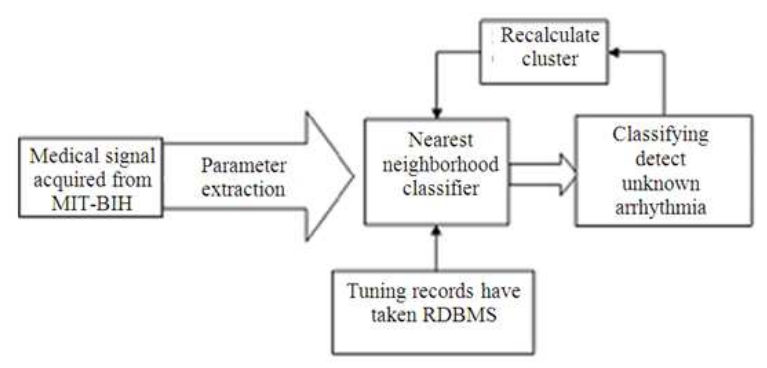

Fig. 2: Testing system model

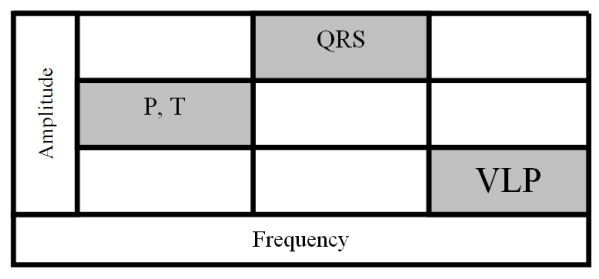

Fig. 3: Frequency distribution of different ECG components

$\mathrm{VM}=\sqrt{\mathrm{X}^{2}+\mathrm{Y}^{2}+\mathrm{Z}^{2}}$ 
Analog to digital conversion: The original continuous analog ECG signal is converted into a digital signal of voltages sampled at frequent, fixed intervals. As with all digitized signals, resolution is governed largely by sampling interval. Sampling rates for VLP evaluations vary from 1000-10,000 samples per second, whereas standard computerized exercise ECG equipment is limited to 250-500 samples sec.

Noise: Numerous sources of noise are encountered in highly amplified recordings. Artifact from respiratory muscles is independent of electrical activity arising from the heart and cancels out with signal averaging. Electronic noise arising from the electrodes is lessened with proper skin preparation. Electrical power lines and other nearby electronic equipment can create noise, which can be reduced by using shielding or filters. The greatest reduction of noise is achieved by increasing the number of cycles averaged.

Filtering: Filters have a great effect on the recognition and measurement of VLPs. Most studies use high band pass filters (cutoff ranging from 25-100 $\mathrm{Hz}$ ), which enable higher frequency signals derived from the depolarization phase of the action potential to pass without attenuation while reducing the low-frequency large-amplitude signals originating from the plateau or depolarization phase of the action potential. Most current systems also use a bidirectional digital filter to reduce artifact, but differing results are still reported with the various filters (Orosco and Laciar, 2009).

Averaging: After analog to digital conversion, the signals are averaged. To use signal averaging techniques the waveform must be periodic and have a specific feature, like the $\mathrm{R}$ wave, which can be used as a reference point so that each waveform can be appropriately aligned (Bunluechokchai and Leeudomwong, 2010). Computer template recognition is currently used to align QRS complexes and to reject ectopic and noisy beats. A frequent sampling interval produces a relatively smooth and continuous waveform. The net effect after averaging is an increase in the signal-to-noise ratio. Most systems include 100-400 beats on average, although some average up to 1000 beats.

Proposed algorithm: The detection of VLPs in the ECG signals is carried out in two stages. First, the features are extracted from the ECG by applying the wavelet transform. Secondly, the recognition of VLPs is done using Ant colony optimization.

Feature extraction using wavelet transform: In recent years, the wavelet analysis has been used widely in biomedical researches (Orosco and Laciar, 2009).
The wavelet transform is a linear time-frequency transform which is based on decomposition of a signal using a set of basis functions. These basis functions are scaled and shifted versions of a prototype mother (Subramanian et al., 2009). The WT produces a timefrequency representation of the signal which is a function of time $b$ and scale $a$. The scale can be considered as the inverse of the frequency (Khushaba et al., 2008). The smaller scales bring about a higher resolution in time which is useful to detect VLPs as they are high-frequency, short-duration signals. In this study, the DWT is adopted, using the MATLAB wavelet toolbox. In this study, a set of data containing records for both VT and non-VT myocardial infarction patients is used. Each record is decomposed into a number of time signals with different frequency contents using DWT techniques. The DWT level containing the frequency range of interest in each signal is then used in the analysis.

Since the DWT uses circular convolution during decomposition and reconstruction, edge effects become apparent whenever the detail coefficients are manipulated. To avoid this effect, the baseline of each lead is corrected. This insures that the end points of the signal meet at the same level without altering the content of the signal. Another possible solution to this problem can be to apply the DWT to a mirror signal, which does not affect the resolution of the coefficient sets.

The DWT of each lead is performed prior to calculating the vector magnitude, which insures the elimination of undesirable cross-terms resulting from the multiplication of different frequency components contained in the signals. These undesirable terms result when calculating the vector magnitude using $\mathrm{X}, \mathrm{Y}$ and $\mathrm{Z}$ leads directly. In this method, level-6 of the DWT of each lead is chosen, since it contains the range of frequencies of interest. The chosen levels are used in the Eq. 1, which is defined as the filtered QRS used in the analysis. The aim is to identify VLPs in the different ECG signals which require a good identification of the QRS complex and its end points. In order to find the onset and offset points of the QRS complex, the algorithm estimates the noise level in the signal. The highest levels in the DWT of the three leads are combined to form the noise estimate. A threshold of this noise in the beginning and end of the signal is calculated and used in the determination of the end points. The duration of the QRS is obtained from the difference between the onset and offset points. A forward and backward search for a $5 \mathrm{~m} \mathrm{sec}$ segment ( 11 samples), where the average exceeds mean +3 standard deviation of noise is performed. For best determination, the noise samples are different for onset and offset values. For onset, a $20 \mathrm{~m}$ ( 44 samples) segment is used that begins at about $50 \mathrm{~m} \mathrm{sec}$ before QRS onset (110 samples). For offset 
point, $40 \mathrm{~m} \mathrm{sec}$ (88 samples) segment which begins at 60 $\mathrm{m}$ sec after QRS (132 samples) is used. After estimating the onset and offset of the filtered QRS complex (the QRS complex in the VM signal), three conventional timedomain features can be measured to detect VLPs (Bunluechokchai and Leeudomwong, 2010):

$\mathrm{QRS}_{\mathrm{T}}=$ Duration of the filtered QRS complex (from the onset to the offset)

$\mathrm{D}_{40}=$ Low-amplitude signal duration (from the offset backward to the point where VM reaches the $40 \mu \mathrm{V}$ and remains at or above this voltage for at least $3 \mathrm{~ms}$ )

$\mathrm{V}_{40}=$ Root-mean-square value of the last $40 \mathrm{~ms}$ of the filtered QRS. These values are based on a $40 \mathrm{~Hz}$ filter.

In Fig. 4, a plot of a typical filtered QRS complex and the definition of the conventional time-domain features, introduced above, can be viewed. The criteria to define a VLP positive test are $\mathrm{QRS}_{\mathrm{T}}>105, \mathrm{D}_{40}>38 \mathrm{~m}$ sec and $\mathrm{V}_{40}<20 \mu \mathrm{V}$.

The QRS duration for MI patients with no VT, which is referred to as normal subjects (Orosco and Laciar, 2009), is found as $95 \pm 10 \mathrm{~m} \mathrm{sec}$. The duration is $139 \pm 26 \mathrm{~m} \mathrm{sec}$ for MI patients with VT, which is referred to us abnormal. The last $40 \mathrm{~m} \mathrm{sec}$ in normal subjects is $73.8 \pm 47.7 \mu \mathrm{V}$ and $14.9 \pm 14.4 \mu \mathrm{V}$ for VT. As can be seen, the type of the filter used affects the resultant values. In study, there is no specific filter applied but rather a bank of filters, which provide an added flexibility in the analysis. The desired cutoff frequency is determined by the sampling rate and the combination of levels or the chosen level by itself. The sampling rate is $22 \mathrm{kHz}$, which gives a Nyquist frequency of $1.1 \mathrm{kHz}$ as the highest frequency in the signal.

Table 1 lists the frequency bands associated with the wavelet decomposition at this sampling frequency and a signal length of 512 samples. The chosen level is level-6 that contained the frequency range from $68.75-137.5 \mathrm{~Hz}$ with a bandwidth of $68.75 \mathrm{~Hz}$, which is equivalent to using a high pass filter with cutoff frequency of approximately $70 \mathrm{~Hz}$. This choice of filter cutoff provides an automatic elimination of the 50 or $60 \mathrm{~Hz}$ line frequencies used in USA and the rest of the world. The QRS duration is found as $101.25 \pm 14.64 \mathrm{~m} \mathrm{sec}$ for normal subjects and $142.14 \pm 29.33 \mathrm{~m}$ sec for VT. The low amplitude signal duration in the last $40 \mathrm{~m} \mathrm{sec}$ is $25.93 \pm 6.32 \mu \mathrm{V}$ for normal and $38.86 \pm 3.75 \mu \mathrm{V}$ for $\mathrm{VT}$. Figure 5 shows a normal ventricular signal averaged ECG, SAECG of patients with mild and severe heart failures.

Only the filtered QRS complex is prolonged, (c) A clearly abnormal SAECG recorded in a man with severe heart failure. It can be seen that all the three parameters are abnormal.
Table 1: Frequency bands and Bandwidth associated with various levels of wavelet decomposition

\begin{tabular}{lcr}
\hline $\begin{array}{l}\text { Levels of wavelet } \\
\text { decomposition }\end{array}$ & Frequency content $(\mathrm{Hz})$ & Bandwidth $(\mathrm{Hz})$ \\
\hline 1 & $4.296-0.00000$ & 1.0740 \\
2 & $8.593-4.29600$ & 4.2960 \\
3 & $17.1875-8.5930$ & 8.5930 \\
4 & $34.375-17.1875$ & 17.1875 \\
5 & $68.75-34.37500$ & 34.3750 \\
6 & $137.5-68.750000$ & 68.7500 \\
7 & $275-137.5000000$ & 137.5000 \\
8 & $550-275.0000000$ & 275.0000 \\
9 & $1100-550.0000000$ & 550.0000 \\
\hline
\end{tabular}

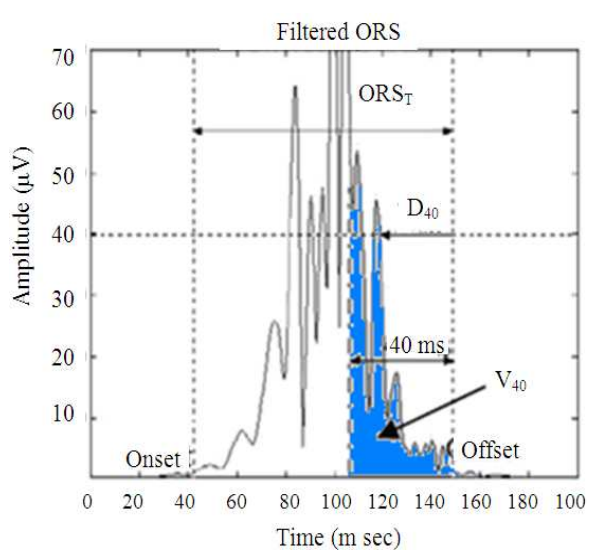

Fig. 4: A ventricular SAECG with the three parameters $\mathrm{QRS}_{\mathrm{T}}, \mathrm{D}_{40}$ and $\mathrm{V}_{40}$ that characterize the filtered QRS complex

For better classification of ECG signals, two more parameters are computed by the WT analysis, in addition to the three standard parameters. These parameters are helpful to improve the performance of classification. The two new parameters are the area (power) under the WT calculated vector magnitude and the norm of the crossterms component resulting from the product of level- 6 and level-5. These two parameters can be called as Wavelet Transform Parameters (WTP). The first of these has a value of $87.31 \pm 14.95$ and the second, $64.94 \pm 42.40$ for normal ECG signals. For VT subjects, the values of these parameters are $117.5 \pm 33.05$ and $43.5 \pm 43.14$ respectively.

Recognition of VLPs with Ant colony optimization: Ant Colony Optimization (ACO) techniques are inspired from the behavior of real ant colonies that are used to solve function or combinatorial optimization problems. Ant colony search algorithms, to some extent; mimic the behavior of real ants. As is well known, real ants are capable of finding the shortest path from food sources to the nest without using visual cues. They are also capable of adapting to changes in the environment; for example, finding a new shortest path once the old one is 
no longer feasible due to a new obstacle. The studies by ethnologists reveal that such capabilities are essentially due to what is called "pheromone trails", which ants use to communicate information among individuals regarding path and to decide where to go. Ants deposit a certain amount of pheromone while walking and each ant probabilistically prefers to follow a direction rich in pheromone rather than a poorer one.

ACO technique can be applied to clustering successfully. The ACO algorithm has been utilized with different favors to solve the clustering problem (Korurek and Nizam, 2008). In this approach's first stage, ants visit other cities randomly and they lay the pheromone according to inverse proportionality of Gaussian distance. After several iterations (cycles), trail intensity (pheromone) between close nodes of trails will be increased; on the other hand, the trail intensity () pheromone) far between the $n$ nodes of trails will be decreased.

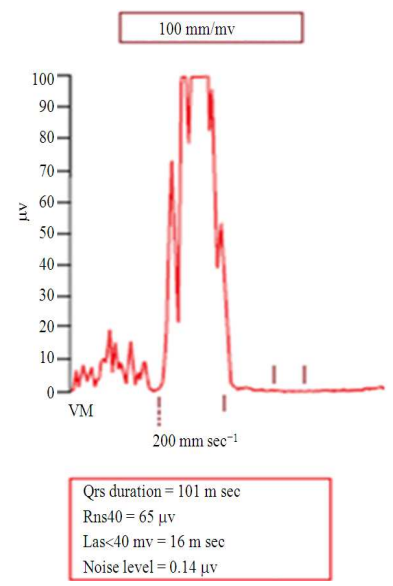

(a)

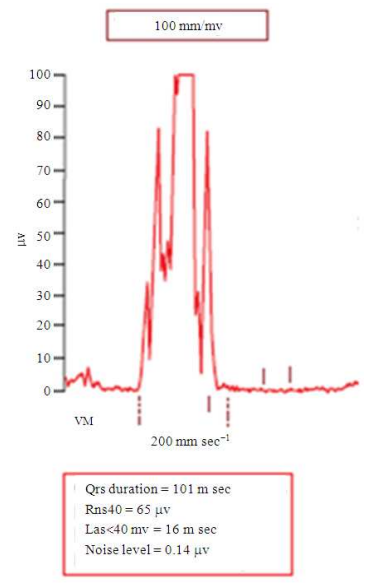

(b)

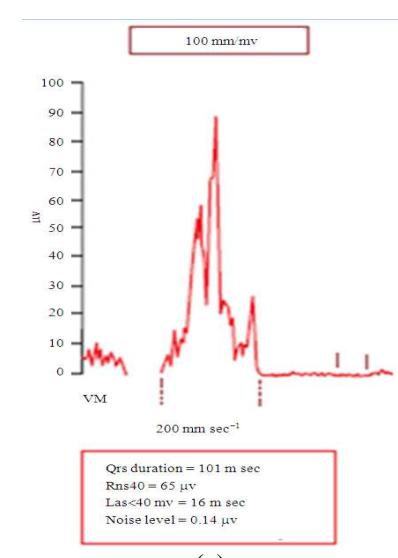

(c)

Fig. 5: (a) Normal ventricular signal averaged ECG, (b) SAECG recorded in a 67 year old man with mild heart failure and no history of arrhythmias
In the second stage, ants will favor to visit the closer nodes and then reinforce the trail with their own pheromone. Every ant only needs to visit (1/10) cities not all of the cities, then the ants decreasingly visit the cities every time. Finally, a number of clans (clusters) will be built.

The tournament selection technique is used for paproportionate selection mechanism and the selection of a new node is based on randomly selected lines is continued as shown in Fig. 6. The choice of the previous path (XS) as a next path is prohibited.

Our study is based on these techniques and some improvements have been implemented. Iteration number is smartly increased and the first and second stages are integrated. Besides, algorithm simplicity is also increased.

The proposed ACO algorithm for clustering can briefly illustrated as follows. The ACO clustering algorithm usually consists of the following steps:

- Initialization: Input $\mathrm{x}$ data sets and assign y ants randomly to $\mathrm{y}$ nodes and initially $\mathrm{y}$ equals $\mathrm{x} / 10$

- Find the candidate nodes the next time for the ants to visit. These nodes are chosen randomly

- Update the pheromone quantity of the visited trail

- Repeat 2 through 5 until the iteration number is reached

- Perform clustering using the value of pheromone quantity

- Each ant visits the other nodes according to the nearest neighborhood interpolation depending on the Gaussian distribution. Select the nearest neighborhood node

Nearest neighborhood classifier: Nearest neighborhood classifier is used for testing purposes. After a new beat is input to the system, the cluster list is scanned to determine the clusters to which it belongs. If there are two or more such clusters, the beat is placed into the cluster which has the maximum number of nearest members. $\mathrm{k}$ is the total number of beat's nearest neighbors and chosen as 5 in study:

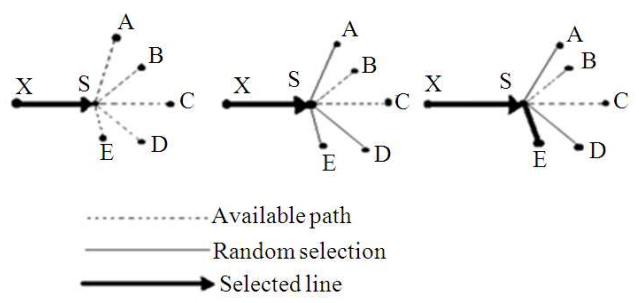

Fig 6: The tournament selection mechanism of ACO algorithm 
Table 2: Results of the classification

\begin{tabular}{|c|c|c|c|c|c|c|c|c|c|c|c|c|c|c|c|}
\hline \multirow[b]{2}{*}{ ECG records } & \multicolumn{5}{|c|}{ WTP } & \multicolumn{5}{|c|}{3 Standard parameters } & \multicolumn{5}{|c|}{$\mathrm{WTP}+3$ Standard parameters } \\
\hline & $\mathrm{TP}$ & FP & FN & $\mathrm{Se}$ & $\mathrm{P}(\%)$ & TP & FP & FN & $\mathrm{Se}$ & $\mathrm{P}(\%)$ & $\mathrm{TP}$ & FP & FN & $\mathrm{Se}$ & $\mathrm{P}(\%)$ \\
\hline Normal & 15 & 02 & 02 & 83.33 & 83.33 & 13 & 05 & 06 & 76.47 & 68.40 & 16 & 01 & 01 & 94.12 & 94.12 \\
\hline VT & 18 & 01 & 03 & 94.70 & 90.00 & 16 & 04 & 05 & 76.19 & 80.00 & 20 & 01 & 00 & 95.24 & 100.0 \\
\hline
\end{tabular}

- Set the member list of the clusters

- Take the next input vector and find its $\mathrm{k}$ nearest members according to the minimum distance measure.

- Find the cluster which the maximum number of nearest members belong

- Assign this cluster as the input vector's cluster

Repeat 2-4 for all input vectors

\section{RESULTS AND DISCUSSION}

As the first step, the WTP parameters are given as input to the Ant colony optimization. The next step is the application of the three standard parameters as the inputs to the ACO the results of the first two steps are close with a slight advantage with the use of WTP. Finally all five parameters are applied to the ACO. In study, an attempt is made to improve the ability to classify the ECG signals originated from different categories of patients. Table 2 summarizes the result obtained. The performance is assessed by the sensitivity and positive productivity, given by the following Eq. 2 and 3:

$$
\begin{aligned}
& \mathrm{Se}=\frac{\mathrm{TP}}{\mathrm{TP}+\mathrm{FN}} \\
& +\mathrm{P}=\frac{\mathrm{TP}}{\mathrm{TP}+\mathrm{FP}}
\end{aligned}
$$

\section{CONCLUSION}

The results clearly show that the combination of WTP and the three standard parameters yields best results for the classification of ECG signals with VLPs. Also the joint use of wavelet transform and the ACO gives an extended capability into the analysis of ventricular late potentials.

\section{REFERENCES}

Bunluechokchai, C. and T. Leeudomwong, 2010. Discrete wavelet transform based base line wandering removal for high resolution electrocardiogram. Int. J. Applied Biomed. Eng., 3: 26-32.
Khushaba, R.N., A. Al sucker, A. Al-Ani and A. Al Jumaily, 2008. Enhanced feature selection algorithm using Ant colony optimization and fuzzy membership. Proceedings of the 6th IASTED International Conference on Biomedical Engineering, (ICBE, 08), ACTA Press Anaheim, USA., pp: 34-39.

Korurek, M. and A. Nizam, 2008. A new arrhythmia clustering technique based on ant colony optimization. J. Biomed. Inform., 41: 874-881. DOI: $10.1016 /$ j.jbi.2008.01.014

Mozus, I., M. Hencu, C. Serban, A. Tudor and L. Susan, 2011. Late ventricular potentials can be predicted from twelve lead ECG in post-infarction heart failure. Int. J. Collaborative Res. Intend Med. Public Health, 3: 53-63.

Orosco, L.L. and E. Laciar, 2007. Bivariable analysis of ventricular late potentials in high resolution ECG records. J. Phy. Con. Ser., 90:1-8. DOI: 10.1088/1742-6596/90/1/012076

Orosco, L.L. and E. Laciar, 2009. Analysis of ventricular late potentials in high resolution ECG records by time-frequency representations. Latin Am. Applied Res., 39: 255-260.

Sivakumar, R., B. Muralikumar, B. Saranya and V.P. Shanthipriya, 2011. Analysis of ventricular late potentials using wavelets and FFT spectrum. Int. J. Biomed. Eng. Technol. 6: 76-92. DOI: 10.1504/IJBET.2011.040454

Subramanian, S.S.A., G. Gurusamy and G. Selvakumar, 2009. Wavelet based detection of ventricular arrhythmias with neural networks classifier. JBiSE, 2: 439-444. DOI: 10.4236/jbise.2009.26064

Tirtom, H., M. Engin and E.Z. Engin, 2008. Enhancement of time frequency properties of ECG for detecting micropotentials by wavelet transform method. Expert Syst. Applied, 34: 746-753. DOI: 10.1016/J.ESWA.2006.10.009

Zandi, A.S. and M.H. Moradi, 2006. Quantitative evaluation of a wavelet based method in ventricular late potential detection. Patt. Recog., 1369-1379. DOI: $10.1016 /$ j.patcog.2006.01.012 